REGARDS

SUR L'ECONOMIE ALLEMANDE

BULLETIN ECONOMIQUE DU CRAC

\section{Regards sur l'économie allemande}

Bulletin économique du CIRAC

$90 \mid 2009$

Varia

\title{
Le facteur humain au cœur du réseau bavarois Sensorik
}

Hubert Steigerwald et Solène Hazouard

\section{OpenEdition}

Édition électronique

URL : http://journals.openedition.org/rea/3573

DOI : 10.4000/rea.3573

ISBN : 978-2-8218-0876-8

ISSN : 1965-0787

Éditeur

CIRAC

Édition imprimée

Date de publication : 1 mars 2009

Pagination : 15-22

ISSN : 1156-8992

Référence électronique

Hubert Steigerwald et Solène Hazouard, "Le facteur humain au cœur du réseau bavarois Sensorik », Regards sur l'économie allemande [En ligne], 90 | mars 2009, mis en ligne le 01 mars 2011, consulté le 19 avril 2019. URL : http://journals.openedition.org/rea/3573 ; DOI : 10.4000/rea.3573 


\section{Le facteur humain au cœur du réseau bavarois Sensorik}

\section{Un entretien avec Hubert Steigerwald}

Avec $30 \%$ de parts de marché et un chiffre d'affaires global, tous acteurs confondus, de près de 30 milliards $€$ en 2007, l'Allemagne est aujourd'hui leader mondial dans le domaine des capteurs de mesure et de commande employés dans l'automobile, les sciences du vivant ou les technologies de l'environnement. L'activité se concentre dans le sud du pays, notamment dans le Land de Bavière qui rassemble près d'un quart des entreprises du secteur. Certaines font partie des 35 membres et 75 partenaires du réseau Sensorik, implanté dans le BioPark de Ratisbonne. Visant à stimuler les transferts de technologies, la création d'entreprises et la formation de personnel qualifié, le cluster labellisé "réseau de compétences » (Kompetenznetz) par le ministère fédéral de l'Economie en 2007 figure parmi les lauréats du concours "Réseaux de compétences 2009 - Pour un développement pérenne des réseaux ". Il a placé l'individu au cœur de sa stratégie. Ce choix s'est révélé judicieux, comme en témoignent les résultats encourageants des premières évaluations menées en 2008, après seulement deux années d'existence.

Dès lors, peut-on s'inspirer de cet exemple de réussite de ce côté-ci du Rhin, où 35 projets de plates-formes d'innovation, aux fonctions équivalentes à celles du réseau allemand, ont été présélectionnés par l'Etat et la Caisse des Dépôts le 5 février dernier dans le cadre de la politique française des pôles de compétitivité ? Afin de comprendre la genèse et les axes stratégiques de Sensorik, qui compte parmi les 2017 clusters recensés par l'Observatoire européen des clusters, nous nous sommes adressés à son manager, Hubert Steigerwald, également directeur du Partenariat Stratégique Sensorik et de la Sensorik-Bayern $\mathrm{GmbH}$. (SH)

Vous avez assisté aux différentes étapes de la constitution du cluster bavarois Sensorik. Pourquoi la région de Ratisbonne?

Cette région, située à proximité de Munich, se caractérise par une forte concentration d'entreprises dans le domaine des capteurs de mesure et d'asservissement (Sensorik). Le service d'expansion économique de la ville de Ratisbonne, ayant saisi le potentiel de ces entreprises en matière d'attractivité territoriale, avait initié dès juin 2003 un partenariat stratégique : à l'époque, 14 responsables économiques et académiques de la ville et du district se sont rassemblés dans une perspective de coopération. De nombreuses réunions se sont tenues, parfois en comité très restreint, jusqu'à ce que le cœur initial du réseau finisse par s'étendre : en août 2006, Sensorik comptait déjà 23 membres...

\section{Comment s'est faite la transition d'un partenariat vers une organisation plus formelle ?}

Une nouvelle impulsion a été donnée en février 2006 par le ministère bavarois de l'Economie, des Infrastructures, des Transports et des Technologies (StMWIVT), qui a fait de notre secteur d'activité l'un des domaines technologiques clés de son programme de promotion des clusters. Notre partenariat stratégique s'est alors doté d'un statut juridique avec la création, le 11 mai 2006, d'une association à but non lucratif qui, dans les mois qui ont suivi, a endossé le rôle de plate-forme bavaroise dans notre domaine : la Strategische Partnerschaft Sensorik e.V. Cette formalisation juridique a permis de percevoir les fonds alloués par le ministère bavarois de l'Economie et d'enregistrer les cotisations des membres, dont le montant pouvait

Un partenariat stratégique s'est organisé autour d'acteurs préexistants... ...puis a été institutionnalisé en 2007 
parfois atteindre $10000 €$. Puis en juillet 2007, nous avons décidé de constituer une SARL, la Sensorik-Bayern GmbH. Pour deux raisons : les acteurs économiques étaient de plus en plus nombreux à souhaiter que nous accomplissions des prestations de services, et le statut de SARL nous autorisait, contrairement à celui d'association, à réaliser des bénéfices. Le cluster s'est ainsi développé dans la continuité.

\section{L'Allemagne, leader mondial dans le domaine des capteurs}

Qu'ils possèdent des propriétés mécaniques, électriques, chimiques, biochimiques ou optiques, les capteurs sont intégrés à des systèmes de réglage et de commande. Agissant comme une véritable interface avec leur environnement, ils peuvent notamment contribuer à déterminer le taux de sucre dans le sang dans le cadre d'un traitement ciblé du diabète, à allier une utilisation réduite de carburant à une meilleure performance automobile, à améliorer la qualité des processus industriels et à en réduire le coût ou encore à localiser une explosion nucléaire à n'importe quel endroit de la planète. II s'agit par conséquent d'une technologie clé aux interconnexions multiples : technique automobile, sciences du vivant, technologies de l'environnement, automatisation, mécatronique...

En 2007, le secteur des capteurs de mesure et d'asservissement a réalisé 22 milliards $€$ de chiffre d'affaires outre-Rhin. Affichant une croissance annuelle de $6,5 \%$ à $12,5 \%$ sur la période 2004-2007, il consacre non moins de $40 \%$ de sa production à l'export et se compose essentiellement de PME : plus de la moitié des entreprises du secteur emploient moins de 50 personnes, $83 \%$ moins de 250 . Le pays dénombre 900 producteurs de capteurs de réglage et de commande. Si on inclut les prestataires de services dédiés, les bureaux d'étude ainsi que les agents commerciaux indépendants, ce chiffre s'élève à près de 2000 , pour un total de 230000 à 250000 collaborateurs. L'Allemagne est leader mondial du secteur avec $30 \%$ de parts de marché. La Bavière y occupe le premier rang en termes de $\mathrm{CA}$ et d'implantation, rassemblant sur son territoire près d'un quart des entreprises. A l'échelle fédérale, les intérêts de la branche sont représentés par la fédération AMA (Arbeitsgemeinschaft Messwertaufnehmer e.V.) : fondée en 1980, elle compte aujourd'hui près de 450 membres, parmi lesquels 70 centres de recherche. Elle publie régulièrement des statistiques de marché, s'implique dans la formation continue et organise des manifestations comme le salon annuel Sensor + Test de Nuremberg (www.ama-sensorik.de). (SH)

\section{En quoi consistait le programme du ministère bavarois de l'Economie ?}

L'innovation, facteur d'emploi Dans le cadre de cette politique, le ministère bavarois de l'Economie accorde à 19 clusters une aide de 50 millions $€$ au total répartie sur cinq ans (2006-2011), Sensorik s'étant vu attribuer pour sa part 1,2 million $€$. Le Land souhaite ainsi renforcer l'attractivité du site économique de la Bavière, où les grandes entreprises industrielles ont bien souvent fait le choix de délocaliser. Par conséquent, l'initiative vise plus spécifiquement les PME, plus fortement ancrées à l'échelle locale et régionale. A travers le soutien à des domaines technologiques clés, elle a pour vocation de stimuler l'innovation, condition sine qua non du maintien et du développement de l'emploi dans la région.

\section{Allianz Bayern Innovativ : l'Alliance pour l'Innovation en Bavière}

Avec l'Alliance pour l'Innovation, le gouvernement de Bavière s'est doté d'un instrument visant à stimuler la compétitivité territoriale du Land. II s'agit dans un premier temps d'y développer, via la Cluster-Offensive, les réseaux de coopération entre universités, centres de recherche, entreprises, prestataires de services et capital-risqueurs dans 19 secteurs clés et/ou domaines de hautes technologies, selon trois objectifs : transposer plus rapidement les résultats de la recherche académique dans le monde de l'entreprise sous la forme de nouveaux produits ou procédés, intensifier la coopération entre les entreprises et leurs fournisseurs locaux tout au long de la chaîne de valeur et enfin consolider l'attachement des entreprises au site économique bavarois. En partenariat avec le réseau dédié à l'électronique de puissance (Cluster Leistungselektronik), Sensorik s'inscrit dans l'un des 19 thèmes d'avenir de ce programme de promotion des clusters. Outre un soutien ciblé par secteur d'activité, l'Alliance pour l'Innovation a pour vocation d'encourager et de renforcer des potentiels de croissance endogènes dans chaque région constitutive du Land. (SH)

L’autonomie comme finalité

Assurer le leadership de la Bavière dans le domaine des capteurs
Comment s'organise la répartition des fonds alloués dans le cadre de cette politique ?

Ce programme incite à la création de plates-formes de coordination de l'activité des réseaux, dont le pilotage stratégique est assuré par un porte-parole, tandis que la gestion opérationnelle est aux mains d'un manager et de son équipe. Chaque manager détermine pour son cluster des objectifs, parmi lesquels celui de l'autonomie financière car les moyens attribués dans le cadre de la ClusterOffensive, constituant au départ entre $80 \%$ et $100 \%$ du capital, diminuent progressivement pour se réduire à terme à $35 \%$. Pour notre part, nous avons pour ambition d'accéder à l'autonomie en l'espace de trois ans, via la rétribution de nos prestations de services et l'obtention de financements lors de manifestations.

\section{Quels sont les objectifs que vous avez définis pour votre cluster ?}

Fort de mon expérience passée dans les activités opérationnelles des groupes Siemens et EADS, j'ai insisté sur la nécessité de fixer des objectifs précis lors de la candidature de Sensorik au programme du ministère bavarois de l'Economie. Où nous situons-nous actuellement ? Où en serons-nous dans cinq ans ? Notre 
vocation première consiste à garantir et développer à l'échelle mondiale le leadership technologique et commercial de la Bavière en matière de capteurs de mesure et d'asservissement. Cela se décline par toute une série de mesures visant à connecter les entreprises entre elles, accroître le potentiel d'innovation, développer nos compétences, étendre notre notoriété, créer de l'emploi, stimuler la création d'entreprises et disposer d'une main-d'œuvre qualifiée.

\section{Comment cela se traduit-il concrètement pour les PME ?}

Si une grande entreprise possède tous les outils requis en matière d'innovation, il n'en va pas de même pour les PME qui ne disposent pas nécessairement d'un département de R\&D ou d'un réel service du personnel et ne peuvent pas consacrer suffisamment de moyens à la formation initiale ou continue. Nous pouvons apporter une aide non seulement dans ces domaines, mais aussi en termes de financement, de marketing et vente, c'est-à-dire que nous mettons sur pied des demandes de financement, proposons des collaborations lors de salons ou assurons par ailleurs des prises de contacts à l'international via la société Bayern International [créée en 1995 à l'initiative du gouvernement de Bavière pour la promotion du commerce extérieur du Land ; SH].

Notre approche repose sur la création d'une relation de confiance. C'est là que le facteur humain prend toute son importance. Car le réseau est constitué d'institutions anonymes ; or pour les relier entre elles, vous avez besoin des responsables, des partenaires, des personnes. Quand vous disposez d'un réseau personnel, vous savez à qui vous adresser lorsque vous rencontrez un problème. Vous ne vous adressez pas à une institution, mais à la personne que vous connaissez en son sein. Voilà pourquoi le facteur humain est au cœur de notre stratégie. Car la confiance que vous avez dans les personnes impliquées permet de résoudre plus vite vos problèmes. L'une des missions de Sensorik est donc de créer la confiance, c'est-à-dire de tisser des liens entre les personnes. Et comment gagne-t-on la confiance de ses partenaires? Par des rencontres. Quand vous savez qui est assis en face de vous, tout devient alors plus simple et plus rapide.

\section{Mais les PME avec lesquelles vous travaillez sont pourtant concurrentes ?!}

II est capital de créer une relation de confiance pour pouvoir travailler ensemble. Lorsque nous rencontrons les dirigeants de PME pour la première fois, ils ne viennent pas nous exposer leurs projets futurs car ce qu'ils ont en tête constitue un potentiel en termes de chiffres d'affaires qu'ils ne souhaitent pas divulguer. Ils ne se confieront à nous que lorsque nous aurons réussi à instaurer une relation de confiance. Ce processus, qui mûrit pendant six mois voire un an, passe par le tutoiement et par un certain nombre de rencontres, procure à terme un sentiment fort : nous n'avons pas seulement trouvé un partenaire, mais aussi peut-être un ami. L'aspect psychologique est déterminant.

\section{Cela suffit-il en matière de confidentialité ou de protection des droits de propriété intellectuelle?}

Quand un entrepreneur vient nous voir pour que nous l'aidions à lancer un produit ou un service innovant, nous signons d'abord un accord de confidentialité où nous nous engageons à ne pas révéler d'informations à des tiers. Toutes les personnes impliquées dans le projet doivent travailler dans ce sens, de sorte que l'entrepreneur conserve la maîtrise de son idée initiale. Lorsque nous avons plusieurs collaborateurs sur un tel projet, il est nécessaire de déterminer ce qui a trait à la propriété intellectuelle et de s'informer de la situation juridique en matière de brevets. Cela se traite toujours en amont, et plus tôt nous nous y consacrons, mieux nous pouvons éviter les conflits susceptibles de faire échouer le projet. En nous basant sur l'expérience des entreprises, nous cherchons à identifier les risques potentiels.

\section{Outre les entreprises, quels sont vos membres et partenaires?}

Au cœur du réseau, Sensorik stimule les échanges entre les membres issus des milieux industriel et universitaire, les responsables en charge de la formation professionnelle continue, les structures de développement économique à l'échelon
Le facteur humain au cœur du réseau

La confiance scelle le partenariat

Evaluer en amont les risques liés à la propriété intellectuelle

Sensorik coordonne le travail en réseau sur l'ensemble de la chaîne de valeur 
L'association
au centre de l'organisation

Le Cluster Sensorik structure institutionnelle de tête de réseau à l'échelle de la Bavière

Sensorik-Bayern GmbH : un centre de compétences éminent local et régional, le ministère bavarois de l'Economie, les $\mathrm{CCl}$, les chambres de commerce extérieur ou encore les chambres de métiers et de l'artisanat. Bien que notre domaine d'activité se compose en grande partie de PME, le cluster comprend aussi de grandes entreprises dont l'expérience constitue une source d'inspiration. Dans ce contexte, divers acteurs participent à l'effort de R\&D : universités, PME, grandes entreprises disposant de leurs propres départements de R\&D, comme Siemens, Continental, Krones ou EADS, et centres de recherche, notamment les Instituts Fraunhofer de Munich et d'Erlangen. Toute la chaîne de valeur dans la production des capteurs de mesure et d'asservissement est donc ici représentée.

Le réseau Sensorik réunit l'ensemble des acteurs de la chaîne de création de valeur

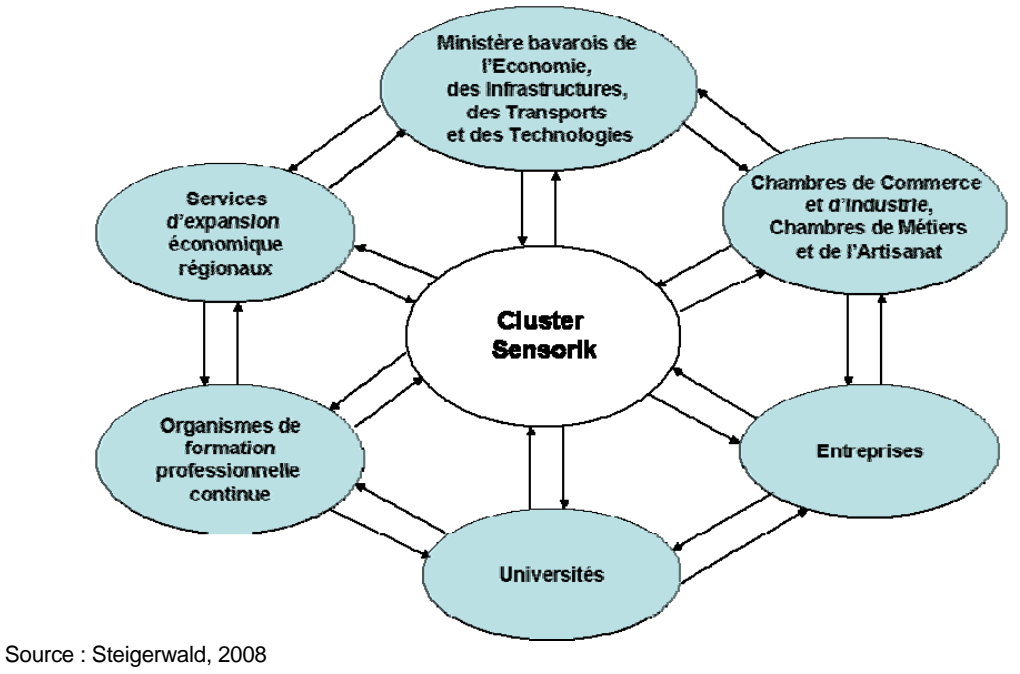

Afin de satisfaire au mieux la demande de vos membres, vous avez donc mis en place une structure institutionnelle reposant sur trois piliers...

Notre structure organisationnelle concorde avec nos missions et objectifs. Le cluster comprend en effet trois entités, avec au centre l'association Strategische Partnerschaft Sensorik e.V. - Partenariat stratégique -, véritable plate-forme de mise en réseau et de suivi des acteurs, en charge des fonds propres et cofinancements. Les membres nous versent des cotisations: les universités contribuent à hauteur de $1000 €$ par an, tandis que certaines entreprises injectent jusqu'à $20000 €$ voire $30000 €$ par an, disposant en contrepartie de certains privilèges comme la réalisation d'études de faisabilité. Outre le financement, l'association se consacre aux ressources humaines, et notamment à la formation professionnelle initiale et continue, avec un pôle ad hoc à destination des membres du réseau et, plus généralement, de toute personne intéressée par les technologies de mesure et de commande.

De l'initiative bavaroise est ensuite née une instance de représentation consacrée au secteur des capteurs...

En effet, la seconde composante, le Cluster Sensorik, a pour mission de représenter les intérêts du Land de Bavière au niveau fédéral et international. L'entité recouvre toute l'activité marketing et l'organisation de salons et foires technologiques. Elle encourage les transferts de technologies et participe à la constitution de dossiers dans le cadre de demandes de soutien financier.

\section{Sensorik-Bayern GmbH constitue le troisième pilier de l'organisation. Quelle est sa fonction au sein du cluster?}

Cette société, qui agit pour ainsi dire comme une filiale de l'association, a été créée par les membres du Partenariat Stratégique. Elle est dédiée à tout ce qui a trait à l'innovation: $R \& D$, études de faisabilité, projets de partenariats, management de l'innovation... La gestion opérationnelle de Sensorik-Bayern $\mathrm{GmbH}$ me revient. II en va de même pour les deux autres entités constitutives du réseau, ce qui crée de 
facto une passerelle entre les instances organisationnelles du cluster Sensorik. Par ailleurs, la composition du conseil d'administration et du comité consultatif de l'association, principal organe de Sensorik, témoigne des bases solides de notre partenariat science/industrie : des dirigeants d'entreprise y côtoient des professeurs d'université, un représentant des collectivités locales, un membre de la chambre de commerce et d'industrie, le dirigeant de la fédération de branche AMA ainsi qu'un représentant de la structure commune de l'Association allemande des ingénieurs et de la Fédération du secteur électrotechnique/électronique (VDI/VDE).

La structure organisationnelle du partenariat stratégique Sensorik

\begin{tabular}{|c|c|c|}
\hline \multirow{3}{*}{ 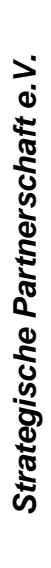 } & Conseil d'administration & $\begin{array}{l}\text { Martin Sellen (Micro Epsilon Messtechnik GmbH \& Co. KG) } \\
\text { Wolfgang Baier (Université de Ratisbonne) } \\
\text { Rudolf Bierl (Continental Automotive AG) } \\
\text { Dieter Dohnal (Maschinenfabrik Reinhausen) } \\
\text { Hermann Jacobs (Infineon Technologies AG) } \\
\text { Toni Lautenschläger (Service d'expansion économique de la ville de Ratisbonne) } \\
\text { Andreas Wolf (Dallmeier electronic GmbH \& Co. KG) }\end{array}$ \\
\hline & Comité consultatif & $\begin{array}{l}\text { Klaus Beumler (VDI/VDE, Porteur de projets) } \\
\text { Richard Brunner (CCI, Réseau de Compétences Mécatronique) } \\
\text { Mikhail Chamonine (Université de Ratisbonne) } \\
\text { Helmut Gesch (Vice-Pdt. de l'Université de Landshut, Cluster Mikrosystemtechnik Landshut) } \\
\text { Hanns Georg Hofhansel (Université de Deggendorf) } \\
\text { Helmuth Hummel (Université de Ratisbonne) } \\
\text { Dirk Rein (Président de la Fédération de branche AMA) } \\
\text { Hans Wolf (Université de Ratisbonne) }\end{array}$ \\
\hline & Gestion opérationnelle & Hubert Steigerwald \\
\hline & Cluster Sensorik & $\begin{array}{l}\text { Porte-parole : Hans Meixner } \\
\text { Manager : Hubert Steigerwald }\end{array}$ \\
\hline \multicolumn{2}{|r|}{ Sensorik-Bayern GmbH } & Directeur : Hubert Steigerwald \\
\hline
\end{tabular}

Source : Steigerwald, 2008

\section{L'association s'occupe donc des questions ayant trait à la gestion du personnel ?}

Comme je l'ai évoqué, le facteur humain est capital : le succès repose sur les individus. Si vous avez des collaborateurs motivés, vous pouvez être assuré de la réussite de votre entreprise. Nous avons donc mis en place un pôle de personnel qualifié regroupant étudiants et personnes en quête d'emploi. Notre objectif n'est pas de mettre les entreprises en concurrence les unes avec les autres. Ces dernières nous exposent leurs besoins pour que nous puissions les aider à embaucher des personnes correspondant parfaitement aux profils recherchés. Comme nous recevons de nombreuses candidatures, nous procédons à des entretiens et réalisons une présélection. Nous aidons par ailleurs le candidat à améliorer la structure de son CV. Ce service créé en 2007 nous a déjà permis de procéder, avec succès, à des recrutements pour le compte d'entreprises. Les personnes nouvellement en poste savent de qui elles tiennent leur emploi, ce qui nous offre un autre type d'accès aux entreprises. Mais cela n'est qu'un élément dans le maillage entre acteurs, qui demeure secondaire par rapport à l'aspect technologique. Le pôle de ressources humaines nous permet par ailleurs d'employer des personnes dont l'entreprise a procédé à des délocalisations. Flexibles, elles peuvent accepter de mettre leurs compétences à disposition des PME sur une base temporaire, ces dernières ne souhaitant pas recruter de tels experts à long terme, mais seulement dans la phase de développement d'un nouveau produit ou d'un nouveau service. II s'agit à la fois de flexibilité pour les PME et de stabilité pour les salariés de notre pôle. La coexistence de la flexibilité et de la stabilité représente aujourd'hui un réel défi.

\section{Que propose le Partenariat Stratégique en matière de formation ?}

Nous voulons éveiller l'intérêt des étudiants pour les technologies de mesure et d'asservissement. Dans cette optique, nous organisons des présentations dans les universités, nous démarchons auprès des étudiants et contribuons à rendre ce domaine de recherche attractif. Nous avons instauré une sorte d'université d'été où

La gestion des ressources humaines est primordiale

Actions de formation destinées à constituer un potentiel de main-d'œuvre 
La SARL en support à la recherche et au transfert de technologies

Un marketing au niveau régional et international...

...en vue d'accroître la notoriété de Sensorik pendant une semaine chaque année, étudiants et collaborateurs d'entreprises sont formés, initiés à la problématique et apprennent mutuellement à se connaître. De grandes sociétés se joignent à cet événement pour évaluer le potentiel en termes de recrutement. Nous regroupons enfin, dans nos académies de formation initiale et continue, les universités du réseau situées dans un rayon de 100 à $120 \mathrm{~km}$.

\section{Pouvez-vous nous préciser les missions incombant à la GmbH ?}

Nous y disposons d'un pôle de compétences dédié à la R\&D dans les technologies de mesure et d'asservissement, où nos propres physiciens, électrotechniciens et biochimistes font de la recherche pour le compte des entreprises. Nos employés savent que nos ressources sont limitées et connaissent leurs interlocuteurs dans les universités bavaroises. Toujours pour le compte des entreprises, nous déterminons le facteur coût et le facteur temps en matière d'innovation, partant du principe que si vous n'êtes pas assez rapide, vous serez vite dépassé par vos concurrents. Ces deux facteurs s'appliquent aussi aux universités, ce qui ne convient pas nécessairement à tout professeur ou à toute unité de recherche. Notre mission est alors de faire en sorte que tous travaillent ensemble dans cette même perspective...

Nous proposons en outre des études de faisabilité : un entrepreneur a une idée qui doit ensuite être formulée dans un dossier de demande de subvention, transmis à un porteur de projets, ici VDI/VDE, avec qui nous sommes régulièrement en contact et qui réalise une présélection. VDI/VDE fait ensuite parvenir la demande à l'échelon concerné (Land, Bund ou UE). Les financements de I'UE ne sont cependant pas adaptés aux besoins des PME car les délais de constitution et de traitement des dossiers sont trop longs pour des entreprises qui, pour survivre, doivent produire de l'innovation à court terme. Ainsi nous n'utilisons les programmes européens que pour les questions relatives aux infrastructures. Nous aidons enfin les PME en matière de management de l'innovation, problématique que nous intégrons par ailleurs à notre offre de formation.

Le Cluster Sensorik, dernière composante du réseau, a quant à lui pour vocation d'accroître la visibilité de votre domaine d'activité, dans une stratégie plus globale de promotion du site économique de la Bavière...

C'est exact. Nous agissons non seulement dans la région de Ratisbonne, de Munich, voire à l'échelle de la Bavière, mais aussi à l'international (Chine, Mexique...), où nous informons les entreprises étrangères des possibilités de collaboration avec notre réseau. Nous organisons des salons, effectuons des présentations en laboratoire, réalisons des actions de marketing pour nos membres, assurons les connexions entre nos membres et mettons sur pied des dossiers de demande d'aide financière. Dans le cadre de salons comme la Hannover Messe ou le salon Sensor + Test de Nuremberg, nous mettons en place des stands communs financés par les cotisations des membres, qui permettent à ces derniers de se rencontrer. Ces stands, toujours bien visités, apportent une meilleure visibilité aux PME. Par exemple, la technologie des capteurs étant très développée dans le secteur automobile, nous avons fait réaliser par nos membres issus de ce secteur une voiture de course équipée de divers capteurs. Présentée lors du salon Sensor + Test de 2008, cette voiture de Formule 1 a retenu l'attention de tous. Elle concentrait les innovations d'EMZ, Hofmann Leiterplatten, Infineon, Micro-Epsilon, PCO. Imaging, PreSens et Siemens VDO. Nous pensons par ailleurs qu'il est dans l'intérêt de notre réseau de prendre part à l'orientation de la politique européenne des clusters : c'est dans cette optique qu'à la fin de l'année 2008, j'ai déposé une candidature en vue d'intégrer le European Cluster Policies Group (ECPG).

\section{Quels sont les résultats de votre stratégie marketing?}

En 2007, nous avons pu rassembler plus de 2000 participants dans le cadre d'une cinquantaine de manifestations. Ces dernières permettent par ailleurs d'obtenir un retour direct des responsables politiques, fortement enclins à promouvoir les technologies de mesure et d'asservissement à l'échelle du Land (stratégiques en matière de communication). Nous organisons enfin des forums technologiques où des responsables d'entreprise, munis d'un cahier des charges, viennent expo- 
ser les difficultés techniques qu'ils rencontrent sur sept ou huit sujets différents en vue d'une coopération. II est important pour nous, en termes de collaboration et de confiance, que les acteurs se réunissent, s'échangent, voire se rencontrent en dehors du travail : ils sont ainsi plus disposés à demander de l'aide à un membre du réseau. Nous réalisons aussi la promotion de notre cluster dans la presse, ce qui nous permet d'améliorer notre notoriété et de recevoir des demandes d'aides supplémentaires de la part d'entreprises.

\section{L'intégration de votre Partenariat Stratégique au programme fédéral des "réseaux de} compétences " (Kompetenznetze) a dû avoir un effet positif en termes de notoriété ?

II s'agit là d'un véritable label en matière de durabilité, de qualité, de transferts technologiques, de promotion de l'innovation et de coopération. Cela nous a permis de communiquer vers l'extérieur et d'asseoir notre présence à l'échelle fédérale. La qualité et la diversité de nos services ont par ailleurs été confirmées par les résultats d'une évaluation menée au premier semestre 2008 par l'Institut Fraunhofer pour la Recherche sur les Systèmes et l'Innovation (ISI) de Karlsruhe pour le compte du ministère bavarois de l'Economie. Le sondage réalisé à cette occasion a donné lieu à un taux de retour exceptionnellement élevé (54\%), ce qui atteste du degré de satisfaction de nos membres, mais aussi du fait que les acteurs se connaissent au sein d'un réseau relativement restreint.

\section{Quelles sont les perspectives d'avenir du cluster?}

Nous entrons dans une phase de consolidation durant laquelle nous allons continuer de nous développer en nous inspirant de ce que nous avons déjà réalisé ces deux dernières années. Dans un premier temps, nous allons mener à bien nos projets actuels car nous voulons prouver qu'à partir d'une idée, nous sommes capables d'aider les entreprises à produire de l'innovation malgré les nombreuses étapes à franchir. L'innovation suppose qu'il existe un besoin commercial : à l'écoute de la demande, nous vérifions constamment l'état et les perspectives du marché ainsi que la situation juridique en matière de brevets. Dans un second temps, nous valorisons nos thèmes de recherche en vue de trouver de nouveaux membres susceptibles de renforcer l'ensemble de la chaîne de valeur. Enfin, nous souhaitons acquérir de nouveaux projets afin de développer l'activité de notre SARL.

\section{La crise économique que nous traversons doit avoir un impact sur les membres de votre réseau, notamment les sous-traitants automobiles...}

La crise financière et économique actuelle pèse, bien sûr, sur certaines entreprises de notre réseau. En raison de la frilosité des banques, certaines, même les mieux gérées, peuvent rencontrer des problèmes passagers de liquidité. Nous y voyons une raison supplémentaire de venir en aide à nos membres et de les soutenir économiquement. Après avoir appréhendé les conditions d'ordre technique et commercial en vigueur dans les entreprises concernées, mon équipe s'attèle activement à la recherche de soutiens publics : elle s'engage notamment à obtenir des subventions au niveau du Land ou de l'Etat fédéral tout en dégageant et en activant des aides complémentaires. Je suis, pour ma part, fortement impliqué dans la promotion des PME à l'échelle fédérale en tant que membre, depuis 2008, du jury du programme ZIM (Zentrales Innovationsprogramm Mittelstand) mis en place par le ministère fédéral de l'Economie. Le recours efficace aux aides publiques permettra à notre organisation et à nos membres de bénéficier, pour l'après-crise, d'un positionnement stratégique sur des projets à forte densité technologique. Car, plus fondamentalement, nous considérons la situation actuelle comme le moment idéal pour innover. Nos membres doivent pouvoir profiter pleinement de notre offre de services. L'approche que nous partageons avec eux consiste à voir dans la « crise » l'opportunité d'aborder de nouveaux domaines technologiques et de jeter les bases d'un avenir serein en termes économiques.

\section{Comment la gestion de crise s'inscrit-elle dans votre stratégie à long terme ?}

Sensorik évolue dans un formidable processus de développement et de croissance, quelque peu à contre-courant de la tendance économique actuelle (ce qui
Un cluster labellisé " réseau de compétences »

Consolider les acquis et la chaîne de valeur

La « crise » : une opportunité pour réaliser de nouvelles conquêtes technologiques

De nouveaux membres et des projets prometteurs 
L'orientation stratégique de Cluster Sensorik: un vecteur de croissance nous réjouit particulièrement en tant que plate-forme de réseau). D'un côté, nous connaissons depuis de nombreuses semaines un afflux exceptionnel de nouveaux membres cotisants (grandes entreprises et PME). De l'autre, à travers ce processus de développement et de croissance, nous nous détachons clairement d'autres réseaux de la Cluster-Offensive. Nous cherchons à nous rapprocher des entreprises en nous orientant clairement vers des projets high-tech pilotes et porteurs d'avenir (capteurs magnétiques innovants pour les systèmes de gestion du trafic, mesures d'épaisseur de couches térahertz ou encore dispositifs de pointage munis d'une caméra dans le domaine de la chirurgie mini-invasive). Par ce biais, nous apportons notre aide aussi bien aux grandes entreprises industrielles bavaroises qu'à de nombreuses PME du secteur qui, grâce à leur offre de niche, parviennent à se hisser au rang de leader mondial. Via des coopérations science/ industrie, notre objectif plus global est de continuer de développer avec succès un site bavarois des capteurs de mesure et de commande au potentiel prometteur.

Notre plate-forme de réseau bénéficie également de ce processus de développement et de croissance, dont la garantie future passe par notre positionnement stratégique en tant que cluster bavarois : en février de cette année, nous nous sommes vu remettre par le secrétaire d'Etat parlementaire auprès du ministère fédéral de l'Economie, Hartmut Schauerte, l'un des prix du concours « Réseaux de compétences 2009 - Pour un développement pérenne des réseaux » qui s'inscrit dans l'initiative «Réseaux de compétences » du ministère. Je souhaite préciser par ailleurs que dans le cadre de l'évaluation menée pour le compte du ministère bavarois de l'Economie, notre cluster a obtenu la meilleure note, aussi bien de la part de notre évaluateur que des membres et acteurs du réseau.

La réussite de l'orientation stratégique de votre réseau semble donc confirmée. Existe-t-il selon vous une recette miracle en matière de clusters?

Bien que notre réseau se développe avec succès suivant le modèle que je vous ai exposé, cet exemple est difficilement transposable. Chaque cluster possède en effet des missions qui lui sont propres et connaît une situation de départ différente. Un seul élément commun demeure cependant : le facteur humain, dont la contribution à la réussite des réseaux est essentielle.

Propos recueillis et traduits par Solène Hazouard

\section{Indications bibliographiques}

- SteigerWALD H., "Strategische Partnerschaft Sensorik e.V. / Bayerisches Cluster Sensorik », Conférence tenue lors du colloque organisé par le CIRAC et le Deutsch-Französisches Institut (Ludwigsburg) en partenariat avec l'ANRT-FutuRIS sur le thème Les défis de l'innovation : stratégies de compétitivité et dynamiques territoriales, à Paris les 9 et 10 octobre 2008

\section{Voir aussi :}

- BALzereit J., Innovationsfaktor Kooperation - Ein Gewinn für Wirtschaft und Wissenschaft!, Chambre de Commerce et d'Industrie de Ratisbonne, octobre 2007

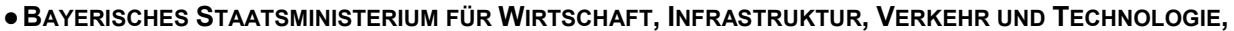
Cluster-Offensive Bayern - Im Netzwerk zum Erfolg, Munich, mai 2008

- BouRGEOIS I., «Bavière : la patiente construction d'une économie performante », in BouRGEOIS I. (dir.), Allemagne : compétitivité et dynamiques territoriales, CIRAC, Cergy-Pontoise, juillet 2007

- "The concept of cluster and cluster policies and their role for competitiveness and innovation: Main statistical results and lessons learned ", SEC(2008) 2637, Europe INNOVA/PRO INNO Europe paper $n^{\circ} 9$, Document de travail des services de la Commission européenne - Annexe à la communication du 17.10.2008 COM(2008) 652 : Vers des clusters de classe mondiale dans I'Union européenne : mise en œuvre d'une stratégie d'innovation élargie

- DOMDEY H., "Le pôle biotech de Munich : une "culture de l'innovation" ", Regards sur l'économie allemande, $n^{\circ} 86 / 08$

- www.allianzbayerninnovativ.de

- www.bayern-international.de

-www.competitivite.gouv.fr

-www.cluster-bayern-leistungselektronik.de

-www.clusterobservatory.eu

- http://cms.isi.fraunhofer.de

-www.kompetenznetze.de

$\bullet$ www.sensorik-bayern.de. 